\title{
Formation and evolution of nuclear star clusters
}

\author{
Alessandra Mastrobuono-Battisti and Hagai B. Perets \\ Physics Department, Technion - Israel Institute of Technology, Haifa 3200003, Israel \\ email: amastrobuono@physics.technion.ac.il
}

\begin{abstract}
Nuclear stellar clusters (NSCs) are dense stellar systems known to exist at the center of most of the galaxies. Some of them host a central massive black hole (MBH). They are though to form through in-situ star formation following the infall of gas to the galactic center and/or because of the infall and merger of several stellar clusters. Here we explore the latter scenario by means of detailed self-consistent $N$-body simulations, proving that a NSC built by the infall and following merger of stellar clusters shows many of the observed features of the Milky Way NSC. We also explore the possibility that the infalling clusters host intermediate mass black holes (IMBHs). Once decayed to the center, the IMBHs act as massive-perturbers accelerating the relaxation of the NSC, filling the loss-cone and boosting the tidal disruption rate of stars up to a value larger than the observational estimates, therefore providing a cumulative constraint on the existence of IMBHs in NSCs. Studying how the properties of the infalling clusters map to the properties of the resulting NSC, we find that, in the IMBHs-free case, the infall mechanism is able to produce many different observational signatures in the form of age segregation.
\end{abstract}

Keywords. galaxies: bulges - galaxies: kinematics and dynamics - galaxies: nuclei - galaxies: star clusters:general - stars: black holes

\section{Introduction}

NSCs are dense massive stellar clusters (with typically a few $\times 10^{6}-10^{7}$ stars), residing in galactic nuclei and most of them hosting a MBH at their center (see Böker 2010, for an overview). They are characterized by effective radii of a few pc and central luminosities up to $\sim 10^{7} \mathrm{~L}_{\odot}$. The formation mechanism of NSCs is still unknown and two are the main competing proposed models: (i) in the in-situ star formation scenario the NSC forms from the gas that migrates to the center of the galaxy (Schödel et al. 2008), (ii) in the cluster-infall scenario massive clusters, like globular clusters (GCs) decay to the center via dynamical friction and merge to form a dense nucleus (Tremaine et al. 1975; Capuzzo-Dolcetta 1993). These two scenarios are not mutually exclusive, and both can contribute to the formation of NSCs. Here we explore the cluster-infall model by means of detailed $N$-body simulations both for the Milky Way (MW) and for generic galaxies. The constituent clusters could host IMBHs (Madau \& Rees 2001; Ebisuzaki et al. 2001; Bromm et al. 2003) and thus we also explore this possibility studying the effects of these massive and compact objects on the formation and evolution of NSCs. In Sect. 2 we describe the initial conditions and the simulations, in Sections 3 and 4 we show and discuss the results obtained.

\section{Simulations and initial conditions}

The models and methods used in our simulations are described in Antonini et al. (2012) and in Mastrobuono-Battisti et al. (2014), where details of the initial conditions of the infalling GCs, as well as of the galaxy model for the background stellar population can be 

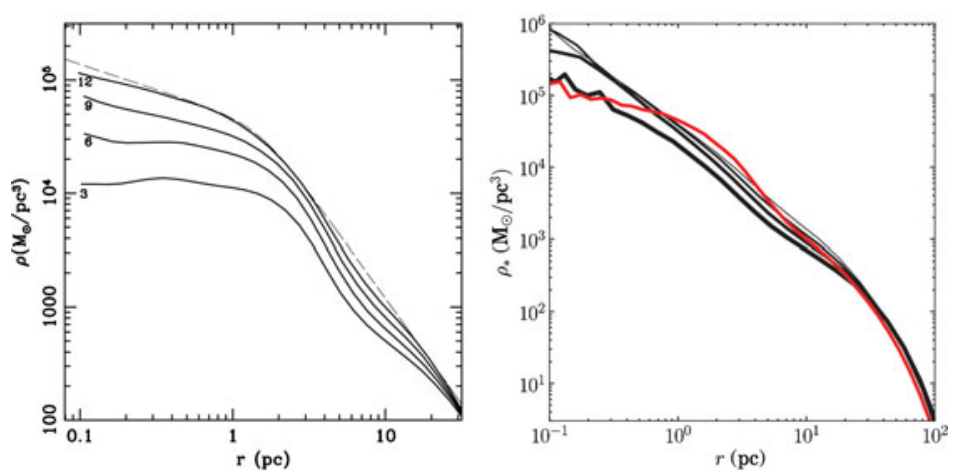

Figure 1. Left panel: Spatial profile of the central NSC after 3, 6, 9 and 12 mergers. The dashed line is the fit to the NSC profile obtained at the end of the last merging event using a broken power law model. Right panel: The same but for the simulation with IMBHs. The thickness of lines decreases with time. The red solid line represents the density profile of the NSC after 12 merging events in the IMBHs-free case. From Antonini et al. (2012) and Mastrobuono-Battisti et al. (2014).

found. In brief, we followed the decay and the merging of 12 massive dense clusters inside the Galactic bulge by means of fully self-consistent $N$-body simulations. The bulge of the simulated galaxy has been modeled based on recent observations (Launhardt et al. 2002) of the MW, while the individual GC $N$-body representation has been done starting from a tidally limited, massive and compact King model. A MBH of $4 \times 10^{6} \mathrm{M}_{\odot}$ is located at the center of the galaxy. We run simulations both with GCs containing only stars and with GCs hosting a central IMBH of $10^{4} \mathrm{M}_{\odot}$. After the first GC decayed to the galactic center, we waited for the NSC to reach a quasi-steady state and we added a second GC to the system. This procedure is iterated until the 12 clusters merged in the inner regions of the galaxy. After the end of all the infalls we followed the relaxation process of the resulting stellar cluster. The total simulation time, rescaled with the relaxation time of the system as described in Mastrobuono-Battisti \& Perets (2013), extends to $\sim 12$ Gyr. We ran our simulations on the GPU partition of the Tamnun cluster at the Technion using $\phi G R A P E$ (Harfst et al. 2007), a direct-summation code optimized for computer clusters accelerated by GRAPE boards (Makino 1998) and recently optimized to run on graphic processing units (GPUs, Gaburov et al. 2009).

\section{Results}

The left panel of Fig. 1 shows the spatial profile of the system, in the IMBHs-free case, after the complete merging of 3,6,9 and 12 clusters. The density grows with time and, at the end of the last merging event, the core radius of the NSC is 4 times larger than the observed value. The merging phase is followed by a two body relaxation which is predicted to shrink the initial core with time, approaching the Bahcall \& Wolf (1976) cusp steady state. The time scale for the process is of the order of the relaxation time at the influence radius of the central $\mathrm{MBH}, r_{\text {infl }}$ (Merritt 2010). After the end of the mergings, the system indeed evolves in a self-similar way, keeping almost unaltered the external radial slope $(\sim-1.8)$ while its core shrinks up to a final size essentially identical to the size of the core observed at the center of the Milky Way $(\sim 0.5 \mathrm{pc}$, see the green line in the left panel of Fig. 2 and Antonini et al. 2012). The NSC evolves toward velocity isotropy (and spherical symmetry) and, at the end of the whole simulation, the NSC has only a small bias toward tangential motion. This is consistent with proper-motion data that indicate a 

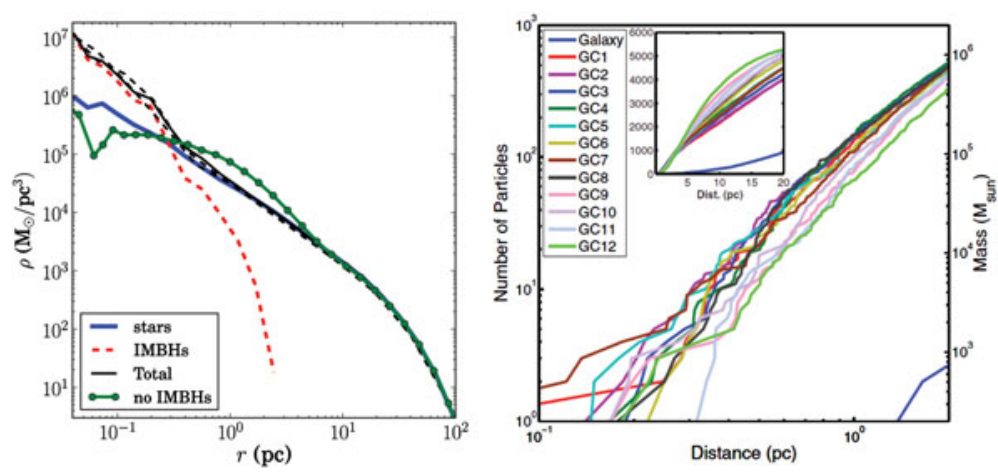

Figure 2. Right panel: The black solid line is the density profile of the NSC at the end of the simulation. The two dashed black lines are the same density profiles obtained from two additional simulations run. The blue solid line shows the spatial profile of the stellar component, while the red dashed line is the final density profile of the IMBHs. The green solid line with bullets shows the density profile of the NSC without IMBHs. Right panel: Radial distribution of the infalling clusters' stellar populations in the case without IMBHs at 12.4 Gyr. Insets show the large scale distribution. From Mastrobuono-Battisti et al. (2014) and Perets \& Mastrobuono-Battisti (2014).

slight degree of tangential anisotropy (Schödel et al. 2014; Merritt 2010). As it is apparent from the right panel of Fig. 1, that shows the density profile of the stellar component of the system after 3, 6, 9 and 12 merging events in the case with IMBHs, the NSC evolution changes significantly when these objects are introduced inside the decaying GCs (Mastrobuono-Battisti et al. 2014). After the host GC is destroyed, each IMBH keeps inspiraling to the center leading to a fast relaxation of the NSC stellar population. At the end of the last infall the inner slope of the radial density profile for the whole system is $\sim-2.1$, steeper then what found in the IMBHs-free case. Indeed, the IMBHs act as massive perturbers, significantly decreasing the relaxation time of the host system (Perets et al. 2007). Therefore, at the end of the simulation, both the stellar cusp and steep radial distribution of the IMBHs are fully developed (see left panel of Fig. 2). The IMBHs cause the system to be in the full loss cone regime at any radius, while without them the loss-cone is empty inside the radius of influence of the $\mathrm{MBH}$. We evaluated the integrated tidal disruption event (TDE) rate using analytic considerations combined with the results of the simulations. Accounting only for stars, we find a rate of $1.6 \times 10^{-5} \mathrm{yr}^{-1}$, while including the IMBHs the rate becomes two order of magnitude higher $\left(10^{-3} \mathrm{yr}^{-1}\right)$. The inferred rate of TDEs from observations is of the order of $10^{-5}-10^{-4} \mathrm{yr}^{-1}$ (see e.g. Khabibullin \& Sazonov 2014) i.e. lower than expected for multiple-IMBHs hosting NSCs such as seen in our simulations. We therefore conclude that typical NSCs should not contain IMBHs. Finally, in order to find signatures left by the merging process in the IMBHs-free case, we explored the mapping between the properties of stellar populations in NSCs, and their relation to the initial characteristics of the stellar populations in the progenitor clusters (Perets \& Mastrobuono-Battisti 2014). We find that stars in the NSC originating from early-infall clusters are more segregated to the center of the NSC than their stellar counterparts from late-infall clusters. This is true both at the end of the merging process and even after few Gyr of evolution (see right panel of Fig. 2). We also find that the three-dimensional structure of the stellar populations of each of the GCs could significantly differ. 


\section{Discussion and conclusions}

Using detailed $N$-body simulations we explored the infall-scenario for the formation and evolution of NSCs. We found that the merging of dense stellar clusters produces a system characterized by a central and almost flat core in the density profile and an external slope of -1.8 , as actually observed in the MW NSC. Moreover the NSC keeps a slight degree of tangential anisotropy matching the observational data (e.g. Schödel et al. 2014). Thus, the process of consecutive infalls and merging in the inner region of the MW of massive dense clusters could provide an explanation for the formation of the Galactic NSC. If present inside the infalling clusters, once decayed to the center, IMBHs are found to scatter each other and the NSC stars, gaining high eccentricities. As a consequence, the NSC develops a cusp already in its early evolutionary stages. The NSC is strongly masssegregated (Alexander \& Hopman 2009) meaning that IMBHs and stars evolve separately and settle on two different profiles characterized by a central cusp with different slopes. The system is in the full loss cone regime and the tidal disruption event (TDE) rates are two orders of magnitude larger than TDE rate estimates from observations, suggesting that typical NSCs, and hence dense stellar clusters, which in the infall scenario are the NSC building blocks, may not contain IMBHs. Moreover, we explored the signatures and the implications of the cluster infall scenario (in the IMBHs-free case), on the structure of NSCs and on their multiple stellar populations. We found that the infall history is reflected in the final structure of the NSC, where stellar populations from earlier infalling clusters are more concentrated in the central parts of the NSC compared to late ones. This dynamical age segregation process can potentially leave behind a signature in the form of an age and/or metallicity radial gradient in the NSC stellar population. In addition, any primordial/early mass segregation in the infalling GCs is mapped into a mass segregated population in the galactic nucleus, potentially leading to efficient mass segregation in NSCs.

\section{References}

Alexander, T., \& Hopman, C. 2009, ApJ, 697, 1861

Antonini, F., Capuzzo-Dolcetta, R., Mastrobuono-Battisti, A., \& Merritt, D. 2012, ApJ, 750, 111

Bahcall, J. N., \& Wolf, R. A. 1976, ApJ, 209, 214

Böker, T. 2010, in IAUS, Vol. 266, IAUS, ed. R. de Grijs \& J. R. D. Lépine, 58-63

Bromm, V., Yoshida, N., \& Hernquist, L. 2003, ApJL, 596, L135

Capuzzo-Dolcetta, R. 1993, ApJ, 415, 616

Ebisuzaki, T., Makino, J., \& Tsuru, T. G., et al. 2001, ApJL, 562, L19

Gaburov, E., Harfst, S., \& Portegies Zwart, S. 2009, NewAstron, 14, 630

Harfst, S., Gualandris, A., \& Merritt, D., et al. 2007, NewAstron, 12, 357

Khabibullin, I., \& Sazonov, S. 2014, MNRAS, 444, 1041

Launhardt, R., Zylka, R., \& Mezger, P. G. 2002, A\&A A, 384, 112

Madau, P., \& Rees, M. J. 2001, ApJL, 551, L27

Makino, J. 1998, Highlights of Astronomy, 11, 597

Mastrobuono-Battisti, A., \& Perets, H. B. 2013, ApJ, 779, 85

Mastrobuono-Battisti, A., Perets, H. B., \& Loeb, A. 2014, ApJ, 796, 40

Merritt, D. 2010, ApJ, 718, 739

Perets, H. B., Hopman, C., \& Alexander, T. 2007, ApJ, 656, 709

Perets, H. B., \& Mastrobuono-Battisti, A. 2014, ApJL, 784, L44

Schödel, R., Feldmeier, A., Neumayer, N., Meyer, L., \& Yelda, S. 2014, ArXiv e-prints

Schödel, R., Merritt, D., \& Eckart, A. 2008, Journal of Physics Conference Series, 131, 012044

Tremaine, S. D., Ostriker, J. P., \& Spitzer, Jr., L. 1975, ApJ, 196, 407 